Meta

Journal des traducteurs

Translators' Journal

\title{
La traduction comme appropriation : le cas des toponymes étrangers
}

\section{Thierry Grass}

Volume 51, numéro 4, décembre 2006

La traduction des noms propres (1) et Langue, traduction et mondialisation : interactions d'hier, interactions d'aujourd'hui Language, Translation and Globalization: Interactions from Yesterday, Interactions from Today (2)

URI : https://id.erudit.org/iderudit/014333ar

DOI : https://doi.org/10.7202/014333ar

Aller au sommaire du numéro

\section{Éditeur(s)}

Les Presses de l'Université de Montréal

ISSN

0026-0452 (imprimé)

1492-1421 (numérique)

Découvrir la revue

Citer cet article

Grass, T. (2006). La traduction comme appropriation : le cas des toponymes étrangers. Meta, 51(4), 660-670. https://doi.org/10.7202/014333ar
Résumé de l'article

Apparemment simple à première vue, la traduction des toponymes pose un certain nombre de problèmes relevant à la fois de la linguistique et de la culture au sens large. Tout d'abord, il n'est pas tout à fait évident de circonscrire ce qu'on appelle un toponyme si ce n'est en lui appliquant le trait [locatif] ; il apparaît alors de nouvelles classes de toponymes comme les objets célestes (der Halleysche Komet = la comète de Halley), les bâtiments (der Pariser Triumphbogen = l'Arc de triomphe) ou les lieux mythiques ou fictifs (Utopia = Utopie) qui ne sont pas celles de l'onomastique traditionnelle. En deuxième lieu, on constate des différences morphosyntaxiques, telle la détermination qui peut être présente en allemand et pas en français ou vice versa (Sachsen = la Saxe; der Mars = Mars). Pour le même toponyme, la référence peut aussi changer (der Genfer See = le lac Léman, der Aralsee = la mer d'Aral et non *le lac d'Aral). S'ajoute à ces phénomènes une dimension qu'on peut qualifier de " poids de l'histoire " : la traduction étant une appropriation, plus un toponyme étranger aura de liens historiques avec une culture donnée, plus on aura tendance à le traduire et inversement. Ceci en dépit des recommandations des Nations Unies en matière de traduction des toponymes.
Ce document est protégé par la loi sur le droit d'auteur. L'utilisation des services d'Érudit (y compris la reproduction) est assujettie à sa politique d'utilisation que vous pouvez consulter en ligne.

https://apropos.erudit.org/fr/usagers/politique-dutilisation/ 


\title{
La traduction comme appropriation: le cas des toponymes étrangers
}

\author{
THIERRY GRASS \\ Université de Tours, Tours, France \\ thierry.grass@univ-tours.fr
}

\begin{abstract}
RÉSUMÉ
Apparemment simple à première vue, la traduction des toponymes pose un certain nombre de problèmes relevant à la fois de la linguistique et de la culture au sens large. Tout d'abord, il n'est pas tout à fait évident de circonscrire ce qu'on appelle un toponyme si ce n'est en lui appliquant le trait [locatif]; il apparaît alors de nouvelles classes de toponymes comme les objets célestes (der Halleysche Komet = la comète de Halley), les bâtiments (der Pariser Triumphbogen = l'Arc de triomphe) ou les lieux mythiques ou fictifs (Utopia = Utopie) qui ne sont pas celles de l'onomastique traditionnelle. En deuxième lieu, on constate des différences morphosyntaxiques, telle la détermination qui peut être présente en allemand et pas en français ou vice versa (Sachsen = la Saxe; der Mars = Mars). Pour le même toponyme, la référence peut aussi changer (der Genfer See = le lac Léman, der Aralsee = la mer d'Aral et non *le lac d'Aral). S'ajoute à ces phénomènes une dimension qu'on peut qualifier de «poids de l'histoire »: la traduction étant une appropriation, plus un toponyme étranger aura de liens historiques avec une culture donnée, plus on aura tendance à le traduire et inversement. Ceci en dépit des recommandations des Nations Unies en matière de traduction des toponymes.
\end{abstract}

\begin{abstract}
Even if the translation of toponyms appears to be simple on a first approach, it can be difficult on a linguistic level as well as on a more general "cultural" level. First, it is not easy to define what a toponym is; it possesses only a [locative] semantic feature. There are classes of toponyms like celestial objects (der Halleysche Komet = la comète de Halley), buildings (der Pariser Triumphbogen = l'Arc de triomphe) as well as mythical or fictive places $($ Utopia $=$ Utopie $)$ that all differ from the traditional classes of onomastics. Second, there are morphosyntactical differences as determination which can appear in German and not in French and vice versa (Sachsen $=$ la Saxe; der Mars = Mars). For a same toponym, the reference can change (der Genfer See = le lac Léman, der Aralsee = la mer d'Aral and not *le lac d'Aral). There is also an aspect which is eligible as "the effect of history": Translation means adapting a name to your own. The more a foreign toponym has contacts to a given culture, the more it will be translated; despite of the recommendations of the United Nations in matters of translation of toponyms.
\end{abstract}

\section{MOTS-CLÉS-KEYWORDS}

globalisation des échanges, Nations Unies, noms géographiques, standardisation, toponyme

Aujourd'hui, à l'heure de la globalisation des échanges ou de la prétendue mondialisation, la tendance vise à une standardisation des noms géographiques pour une soi-disant meilleure compréhension internationale. Cette standardisation, en ce qui concerne les langues française et allemande, est opérée sous l'égide des Nations Unies par le GENUNG (Groupe d'experts des Nations Unies pour l'Uniformisation des Noms 
Géographiques) et par le StAGN (Ständiger Ausschuss für Geographische Namen), mais elle n'est pas toujours suivie d'effets. De fait, ce qu'on peut appeler «le poids de l'histoire» joue un rôle qu'on ne saurait sous-estimer, si bien que le Chinois de Königsberg ne deviendra jamais le Chinois de Kaliningrad. Cela dit, des choix qui défient parfois l'intelligibilité sont parfois opérés dans des publications reconnues et l'atlas allemand Grosser Atlas der Welt fait par exemple état du Zatoka Pomorska (Golfe de Poméranie) sans qu'il soit jamais question de Pommersche Bucht, alors que les eaux de ce golfe touchent aussi l'Allemagne. Nous montrerons dans cette contribution que si la traduction des toponymes étrangers ou exonymes tend souvent à une appropriation, leur dénomination se teinte d'idéologie à l'encontre de toute systématique, au hasard des alliances, de ce qui est sensé être "politiquement correct»ou, au contraire, des inimitiés géopolitiques. Dans le cadre du projet Prolex de base de données lexicale multilingue de noms propres, certains principes prévalent pour entrer des toponymes et leurs traductions, et nous verrons que les normes édictées par les Nations Unies entrent parfois en conflit avec les usages historiques. Nous nous attacherons enfin à des considérations plus linguistiques en mentionnant les informations minimales devant figurer dans un dictionnaire bilingue des toponymes.

\section{Toponymes et traduction}

Pour Denis Le Pesant du Laboratoire de Linguistique Informatique ${ }^{1}$, les noms locatifs «constituent une catégorie de noms d'objets dimensionnels, tels que leurs méronymes d'espace ont pour hypéronyme le mot lieu». Autrement dit, les toponymes entretiennent des relations contenant-contenu dans l'espace et sont tous des sortes de lieux. Cette définition a l'avantage de pouvoir inclure parmi les toponymes des espaces définis par des superficies comme les villes, les régions ou les pays; des espaces déterminés par des tracés comme les cours d'eaux; des espaces en mouvement comme les astres; et même des espaces fictifs comme l'Atlantide engloutie. Dans la base de données Prolex, nous avons 10 types de toponymes qui correspondent en partie à des «classes d'objets» lexico-syntaxiques telles que celles-ci ont été définies par le linguiste Gaston Gross. Les classes Prolex sont destinées en priorité au traitement automatique et pas à une consultation humaine; néanmoins, nous avons voulu utiliser une terminologie qui n'exclut pas la consultation humaine. La perpective retenue par Prolex n'est toutefois ni encyclopédique ni diachronique.

\subsection{La classe des toponymes dans Prolex}

Le projet Prolex (Piton, Grass et Maurel 2003), basé à Tours, a pour objectif de créer des outils permettant le traitement automatique des noms propres. Le noyau de ce projet est constitué par une base de données de noms propres traduits dans plusieurs langues, dont l'allemand. La base différencie 10 types de toponymes que nous allons brièvement énumérer :

1. Les noms de pays (Frankreich = la France, die Schweiz = la Suisse).

2. Les noms de régions où région doit être compris comme une subdivision d'un pays (Steiermark = la Styrie, die Westindischen Inseln = les Antilles).

3. Les noms de groupes de pays, des ensembles englobant différents pays (der Balkan = les Balkans). Les groupes de pays ne doivent pas être confondus avec des régions qui sont des méronymes de pays alors que les pays peuvent être des méronymes de groupes de pays. 
4. Les noms de villes, comme Tours, Paris ou Munich. Ce dernier est un exonyme, c'est-àdire une forme locale du nom de la ville (München).

5. Les noms de quartiers, de voies ou de places (Kreuzberg, Goethestrasse, Savignyplatz).

6. Les noms d'édifices incluent non seulement les bâtiments, les monuments, les ponts, mais aussi les parcs et jardins, les musées, les théâtres et opéras, etc. (der Stift in Frankfurt, der Englische Garten in München, die Wiener Staatsoper). Ils sont souvent traduits car ils sont, pour la plupart, descriptifs (der Stift = le "crayon», der Englische Garten = le Jardin anglais, die Wiener Staatsoper = Opéra national de Vienne).

7. Les hydronymes comprennent les noms de rivières, de canaux ainsi que les différentes étendues d'eau comme les lacs, les mers, etc. (der Rhein = le Rhin, der Bodensee = le lac de Constance, das Mittelmeer = la Mer Méditerrannée).

8. Les géonymes sont des sites géographiques naturels qui incluent les déserts, les montagnes, les forêts, les cavernes, les glaciers, les canyons, les plaines, les plateaux, les courants marins, etc. (die Wüste Gobi = le désert de Gobi, der Schwarzwald = la Forêt-Noire).

9. Les objets célestes représentent - du moins dans la langue - des lieux dans l'espace. Les objets célestes comprennent les planètes, les galaxies, les étoiles, les comètes, etc. (der Saturn $=$ Saturne, Andromeda $=$ Andromède, der Hundstern $=$ Sirius $)$.

10. Les noms de lieux mythiques ou fictifs, bien que conceptuellement imaginaires, ont une syntaxe qui les rattache à une classe d'objets particulière: pays, île, rivière $($ Utopia $=$ Utopie, Atlantis $=$ l'Atlantide, $\operatorname{der}$ Styx $=$ le Styx $)$.

\subsection{Procédés de translation des toponymes}

La translation des toponymes constitue le passage d'une langue à une autre sans être nécessairement accompagné d'un changement de forme graphique. La translation recouvre quatre cas de figure selon que le nom propre reste inchangé, qu'il est transcrit, translittéré ou traduit. Des combinaisons de ces différents procédés sont aussi possibles. De plus, la forme obtenue par translation interagit avec les caractéristiques morphologiques de la langue cible. Le toponyme étranger, par rapport au français, est soit une forme locale ou endonyme qui est empruntée comme Appenzell, Bregenz, Prater ou Oder, soit une forme traduite appelée exonyme comme Autriche, Saxe, Munich, Porte de Brandebourg ou lac de Constance. Notons que dans la grande majorité des cas, la traduction d'un nom propre est un emprunt.

L'emprunt est déjà défini dans la Stylistique comparée du français et de l'allemand de Malblanc dans une catégorisation des procédés de traduction toujours actuelle (1968: 26-30), il consiste au sens strict à importer le mot tel quel, sans rien changer à la structure graphique $($ Donaueschingen $=$ Donaueschingen $)$.

C’est simple à première vue, mais langue source et langue cible n'ont pas toujours le même alphabet: Москва translittéré Moskva est traduit par Moskau en allemand et Moscou en français. On ne peut pas parler d'emprunt proprement dit pour Moskva du fait qu'il y a changement de la structure graphique en passant de l'alphabet cyrillique à l'alphabet latin; dans Moskau et Moscou, il y a non seulement changement de structure graphique, mais aussi changement de structure phonique dans le passage du russe au français et du russe à l'allemand. Il y a donc deux stratégies pour intégrer un toponyme écrit dans un alphabet ou selon un système graphique différent de l'alphabet latin, la translittération et la transcription.

La translittération fait correspondre en théorie à chaque signe d'un système d'écriture un signe dans un autre système, rien ne se perd et la translittération a pour 
avantage principal d'être réversible. Pourtant, la translittération présente un gros désavantage: comme elle est ancrée à la langue étrangère et non à celle qui importe le toponyme, elle peut se révéler partiellement illisible comme Čel’abinsk, forme translittérée selon la norme ISO de la ville de Tcheliabinsk dans l'Oural (en allemand Tscheljabinsk). Pour pallier les désavantages inhérents à la translittération, on a recours à une transcription.

La transcription est l'adaptation de l'image phonique du toponyme étranger à la norme graphique endogène et à chaque phonème correspondent un ou plusieurs signes. L'avantage principal de la transcription est d'être ancrée dans la langue cible, ce qui permet une plus grande lisibilité et un semblant de prononciation. En revanche, du fait qu'elle n'intègre pas la totalité des graphèmes de la langue source, la transcription est irréversible, d'où les variations selon les langues et les formes différentes des toponymes non écrits en alphabet latin en français et en allemand notamment. Si Tcheliabinsk correspond à la transcription française, Tscheljabinsk correspond à une transcription allemande et il n'est pas possible de déterminer des règles rigoureuses permettant de passer d'une forme à l'autre, ou de revenir à la forme originale en alphabet cyrillique.

\section{La traduction}

Chaque langue note les toponymes d'une autre langue selon un certain usage qui existe parfois depuis des siècles et qui dépend en grande partie de facteurs culturels que nous ne développerons pas ici. Pour reprendre la terminologie de Malblanc, nous verrons deux procédés de traduction au sens strict courants en toponymie, le calque et l'adaptation.

Le calque constitue une traduction littérale comme dans White House $=$ Weißes Haus, Maison-Blanche. La notion de calque dépend évidemment du couple de langues choisi. Par exemple, dans le cadre de la traduction anglais-allemand, Strasse von Dover est un calque de Straits of Dover, dont il reproduit la structure lexico-syntaxique; en revanche, dans le cadre de la traduction français-allemand, Strasse von Dover n'est pas un calque de pas de Calais.

L'adaptation constitue une appropriation linguistique, elle peut être phonétique quand il y a changement de la représentation graphique de l'image phonique du toponyme, c'est notamment le cas pour la francisation des noms alsaciens (ex.: Zabern $=$ Saverne) ou de la germanisation de toponymes polonais comme Wroclaw prononcé [' $v r c t s w a f]$ qui devient Breslau. Mais l'adaptation peut aussi être sémantique lorsque le référent (der Genfer See = le lac Léman) ou la structure conceptuelle du toponyme changent (der Aralsee = la mer d'Aral et non ${ }^{*}$ le lac d'Aral).

\section{La tentative de standardisation des toponymes}

\subsection{Les Nations Unies et le traitement des noms géographiques}

Le Groupe d'experts des Nations Unies pour les noms géographiques (GENUNG) a pour vocation de normaliser l'orthographe des toponymes pour que les désignations des noms de villes, de villages, d'étendues de terre et de cours d'eau soient claires, précises, actualisées et sans ambiguïté. Dans une société globalisée, l'efficacité des communications dépendrait en partie de l'utilisation correcte de ces noms sur les cartes 
géographiques, dans les médias et dans les documents juridiques. Des recommandations édictées par les Nations Unies, on retiendra que pour les États qui utilisent l'alphabet latin, on doit utiliser la nomenclature officielle publiée par l'État en question en respectant fidèlement les signes diacritiques.

Pour les États ayant d'autres systèmes d'écriture, on a soit recours au système officiel de translittération de ces États, s'il existe, soit à une orthographe internationale reconnue par les États en question. Cependant, certains pays comme la Suisse ont plusieurs langues officielles et au sein d'un même pays peuvent cohabiter plusieurs minorités régionales possédant elles-mêmes leurs langues, ce qui est par exemple le cas de la minorité slovène en Carinthie. Enfin, lorsque la langue nationale n'est pas exprimée dans l'alphabet latin, on se heurte aux problèmes évoqués précédemment relatifs à la transcription ou à la translittération. Toutefois, comme les villes sont incluses dans le territoire d'un État et que les Nations Unies raisonnent en termes d'États, ce n'est pas tellement ces toponymes qui posent des problèmes mais plutôt les zones géographiques partagées entre les États comme les montagnes ou les fleuves. Un exemple souvent cité est celui du Danube, fleuve européen par excellence qui porte les noms de Donau en Allemagne et en Autriche, Duna en Hongrie, Dunav en Croatie, en Yougoslavie et en Bulgarie, Dunărea en Roumanie et Dunaj en Ukraine; le fleuve doit-il sur une carte changer de nom à chaque changement de rive ou passage de frontière? Doit-on renoncer aux exonymes pour être politiquement correct, c'est un choix éditorial qui a été réalisé par certains atlas dont le Grosser Atlas der Welt publié chez Bechtermünz Verlag, c'est un choix aussi en accord avec les principes des Nations Unies, mais l'est-il avec la pratique linguistique?

\subsection{Le GENUNG francophone, vecteur d'uniformisation pour le français}

La Division francophone du GENUNG ${ }^{2}$ a été créée lors de la $7^{\mathrm{e}}$ Conférence sur la normalisation de noms géographiques tenue à New York en janvier 1998.

Lors de cette $7^{\text {e }}$ Conférence, plusieurs pays dont la Belgique, le Bénin, le Cameroun, le Canada, la Côte d'Ivoire, la France, le Laos, le Luxembourg, le Mali, Monaco, la Roumanie et la Suisse ont apporté leur soutien au projet dont l'un des principaux objectifs a été de faire en sorte que l'expertise des pays avancés dans le domaine de la normalisation des noms géographiques puisse servir à l'ensemble de la communauté francophone, et que les pays ne possédant pas encore de structures toponymiques nationales trouvent au sein de cette division un espace approprié à l'expression de leurs besoins spécifiques. Le secrétariat provisoire de la Division est sis auprès de la Commission de toponymie de l'Institut géographique national français. Le GENUNG travaille notamment sur la romanisation des noms géographiques écrits dans d'autres alphabets, la formation en toponymie dans les pays le souhaitant, la mise sur pied de répertoires des noms géographiques dans les pays concernés ainsi qu'une méthodologie pour la collecte des noms géographiques.

Bien que travaillant de concert avec la Commission de toponymie de l'Institut géographique national, la Division francophone du GENUNG ne s'occupe véritablement que des endonymes ou toponymes autochtones. Le traitement des toponymes étrangers pour l'usage français n'a jusqu'à présent pas fait l'objet d'une normalisation nationale, hormis les noms de pays, de capitales et d'habitants, dont les formes françaises officielles ont été fixées par le ministère des Affaires étrangères dans un docu- 
ment de juin 1995 en cours de révision. En tant qu'organisme administratif, l'IGN doit se conformer à cette liste. Pour une petite partie de la toponymie mondiale, le français possède des exonymes, c'est-à-dire les formes françaises, consacrées par l'usage, de noms étrangers. Ces exonymes ont été intégrés dans notre langue selon divers processus qui en ont fait un ensemble extrêmement hétérogène et sans logique interne; ainsi, à partir d'un nom unique russe translittéré Moskva, a-t-on en français deux noms différents, Moscou pour la capitale de la Russie et Moskova pour le fleuve qui y coule.

À cette diversité vient s'ajouter le problème des langues non écrites, ou écrites dans des systèmes non latins (arabe, chinois, cyrillique, grec, etc.). Les premières requièrent des systèmes de transcription, basés sur la prononciation de la langue d'origine, les secondes des systèmes de translittération, qui établissent des correspondances entre les signes graphiques de l'écriture d'origine et l'alphabet latin. Lors de l'établissement d'un dictionnaire bilingue et, a fortiori, multilingue des toponymes, le rédacteur fait donc face à l'existence de deux ou même plusieurs variantes pour un même toponyme et ceci dans chaque langue considérée. Comme une traduction ne peut se satisfaire de solutions de rechange, il faut faire un choix, sachant que celui-ci peut être contesté selon l'école.

\subsection{Le StAGN, vecteur d'uniformisation pour l'allemand}

Le StAGN (Ständiger Ausschuss für Geographische Namen = Comité permanent pour les noms géographiques) est le pendant de la Division francophone du GENUNG. Il travaille sur l'uniformisation de l'emploi officiel et privé des noms géographiques dans le domaine germanophone par l'édition de répertoires, suivant les directives des Nations Unies, ainsi qu'à l'élaboration d'une liste d'exonymes ${ }^{3}$. Le StAGN, en tant qu'institution allemande, travaille aussi en collaboration avec des institutions autrichiennes et helvétiques visant à obtenir une standardisation dans le monde germanophone, ce qui n'est pas tout à fait le cas pour l'emploi de la lettre «ß». La liste d'exonymes d'origine française publiée par le StAGN est suffisamment courte pour trouver sa place ici.

\begin{tabular}{|l|l|l|}
\hline Deutsches Exonym & Endonym & Objekt \\
\hline Burgund & Bourgogne & Gebiet \\
\hline Diedenhofen & Thionville & Stadt \\
\hline Dünkirchen & Dunkerque & Stadt \\
\hline Elsass & Alsace & Gebiet \\
\hline Flandern & Flandre & Gebiet \\
\hline Genfer See & $\begin{array}{l}\text { Lac Léman } \\
\text { Le Léman, Lac de Genève }\end{array}$ & See \\
\hline Gesellschaftsinseln & Îles de la Société & Inseln \\
\hline $\begin{array}{l}\text { Grosser Belchen [CH], } \\
\text { Großer Belchen [AT, DE] } \\
\text { Sulzer Belchen }\end{array}$ & $\begin{array}{l}\text { Grand Ballon } \\
\text { Ballon de Guebwiller }\end{array}$ & Berg \\
\hline Hagenau & Haguenau & Stadt \\
\hline Hennegau & Hainaut & Gebiet \\
\hline Korsika & Corse & Insel \\
\hline
\end{tabular}




\begin{tabular}{|l|l|l|}
\hline Lothringen & Lorraine & Gebiet \\
\hline Maas & Meuse & Fluss \\
\hline Mülhausen & Mulhouse & Stadt \\
\hline Neukaledonien & Nouvelle-Calédonie & Insel \\
\hline Nizza & Nice & Stadt \\
\hline Pyrenäen & Pyrénées & Gebirge \\
\hline Saarburg & Sarrebourg & Stadt \\
\hline Saargemünd & Sarreguemines & Stadt \\
\hline Savoyen & Savoie & Gebiet \\
\hline Schelde & Escaut & Fluss \\
\hline Schlettstadt & Sélestat & Stadt \\
\hline $\begin{array}{l}\text { Strassburg [CH], } \\
\text { Straßburg [AT, DE] }\end{array}$ & Strasbourg & Stadt \\
\hline Vogesen & & \\
\hline $\begin{array}{l}\text { Weissenburg [CH], } \\
\text { Weißenburg [AT, DE] }\end{array}$ & Vosges & Gebirge \\
\hline Zabern & Wissembourg & Stadt \\
\hline Zentralmassiv, Zentralplateau & Massif central & \\
\hline
\end{tabular}

En regard du passé commun et des zones passées sous contrôle à un moment donné de l'histoire, on peut considérer qu'il s'agit là d'une liste très réduite; on remarquera que certains des toponymes consignés dans cette liste ne sont pas proches d'un État germanophone.

\subsection{Le poids de l'histoire dans la traduction des toponymes}

Bien entendu, la dénomination d'un toponyme n'est pas neutre politiquement, comme l'a montré la vague des villes débaptisées dans les pays de l'ancien bloc soviétique ou dans l'ancienne Prusse orientale après les deux guerres mondiales. C'est que les noms de lieux subissent une influence qu'on peut qualifier de "poids de l'histoire»: soit que le lieu change de nationalité et de langue, soit qu'il entretienne des liens historiques puissants avec une culture donnée. La traduction est une appropriation, et les peuples s'approprient les noms des lieux de leur histoire. L'utilisation d'un toponyme plutôt qu'un autre pour le même lieu n'est donc pas gratuite et reflète une époque, une façon de penser et de se représenter le lieu et ses habitants et parfois même un événement: la Bérézina, cet affluent du Dniepr, est devenu synonyme d'échec catastrophique en français; Stalingrad, nom donné de 1925 à 1961 à la ville de Volgograd est marqué par la période du stalinisme; Auschwitz ou Theresienstadt n'ont pas les mêmes connotations ou sèmes afférents qu'O wi cim ou Terezín, villes de Pologne et de République tchèque. Quoi qu'il en soit, il est important de ne jamais perdre de vue le contenu historique du toponyme, c'est là une limite au traitement synchronique dans une base de données lexicale.

Les conquêtes de territoires et la dimension centrifuge du jacobinisme ont eu des répercussions linguistiques dans les régions germanophones de l'est de la France. Le souci des autorités centrales a pendant longtemps, disons jusqu'à la décolonisation, été de donner des formes ou des consonances françaises aux noms propres. Dans le cadre alsacien, comme le souligne Solange Wydmusch (1998:78) : «Partout les termi- 
naisons en weiler sont devenues willer ou viller. Sur les cartes du géographe Cassini en 1812, Hangweiler devient Hangwiller [...]. Mais il existe des exceptions comme pour Weiler près de Wissembourg. [...] Les noms en burg sont devenus bourg, ainsi Lauterbourg, Eschbourg [...].» Ainsi que le mentionne Meininger (1986: 130) dans son excellent Dictionnaire des toponymes et des vieux termes mulhousiens, les variantes Wilre, Wiler ou Weiler signifient village en alsacien, racine qu'on retrouve aussi en patois franc-comtois dans les toponymes en -villers comme Indevillers, Fessevillers ou Burnevillers dans le Haut-Doubs. La francisation, sur laquelle on revient à présent dans les régions à langues régionales comme l'Alsace, en faisant figurer certains toponymes comme les noms de rues dans les deux langues, met en relief la fantaisie des personnes autrefois chargées de la francisation des noms. On constate ainsi que si les noms de rues ont été traduits dans les grandes villes alsaciennes comme Mulhouse, ce n'est pas toujours de façon systématique: si die Wilde Manngasse est devenue rue du Sauvage, die Beckengasse, rue des Boulangers, die breite Strasse quant à elle a été rebaptisée rue Engelmann du nom du graveur mulhousien ayant introduit en France la lithographie. Dans l'ensemble, les noms de monuments comme Bollwerkthurm = tour $\mathrm{du}$ bastion sont fidèlement traduits. Pour ces quelques exemples symptomatiques, il faut insister sur le fait que même si les formes germaniques sont antérieures aux formes françaises, elles ne sont pas réutilisables dans une traduction du français vers l'allemand, sous peine de se faire taxer de pangermanisme. L'usage distingue cependant entre les noms de rues qui ne sont pas traduits, comme la rue du Sauvage qui devient die rue du Sauvage, et les noms de monuments qui sont très souvent traduits, comme le Haut-Konigsbourg qui redevient der Hochkönigsburg. Quant aux noms de villes, il appartient de se référer à la liste d'exonymes du StAGN que nous avons fait figurer ci-dessus.

À l'inverse de ce qui s'est passé entre la France et l'Allemagne, la Suisse a géré de façon plus équilibrée sa situation de quadrilinguisme. C'est en effet le seul pays européen avec la Finlande à tolérer l'emploi des différentes formes des toponymes résultant de la pluralité des langues officielles de façon officielle et indifféremment. Pour la Belgique, pays trilingue, la situation est un peu plus délicate et, contrairement à la Suisse, seuls les toponymes utilisés dans la région même sont employés de façon officielle, ce qui n'empêche pas un grand nombre de traductions de part et d'autre de la frontière linguistique néerlandais-français.

Enfin, il faut dire que les espaces francophone et germanophone se sont en partie constitués l'un aux dépens de l'autre tout au cours de l'histoire, ce qui suppose conquêtes et reconquêtes sur un substrat à l'origine commun. C'est cette tradition historique et une forte interpénétration culturelle qui explique la survivance d'exonymes fort nombreux.

\section{Quelles sont les informations qui doivent figurer dans un dictionnaire électronique bilingue des toponymes?}

Dans la base de données lexicale Prolex, nous avons choisi de faire figurer un certain nombre d'informations permettant non seulement de référencer l'unité lexicale dans une optique de traitement automatique, mais aussi de donner des informations à caractère sémantique. 


\subsection{Genre et nombre}

Les toponymes sont dans la majeure partie des cas au singulier, mais parfois aussi au pluriel. Contrairement à ce qui est le cas pour d'autres noms propres, une forme exclut l'autre, ce qui veut dire qu'un toponyme au singulier n'a pas de pluriel et qu'un toponyme au pluriel n'a pas de singulier:

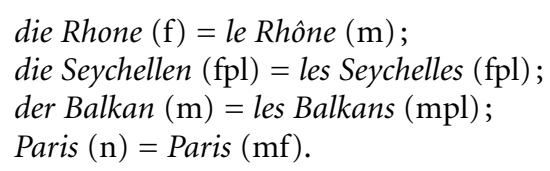

\subsection{Flexion casuelle}

Pour les langues slaves, le nombre de formes fléchies d'un nom propre peut atteindre quelques dizaines. La flexion casuelle, pour ce qui est de l'allemand, ne concerne dans la plupart des cas que la forme du génitif singulier et ceci seulement pour les toponymes masculins et neutres. Pour les noms d'édifices, il en va toutefois différemment et Weißes Haus prend les flexions casuelles de ses composants.

\begin{tabular}{|l|l|l|}
\hline & masculin singulier & neutre singulier \\
\hline Nominatif & der Balkan & das Karwendel \\
\hline Accusatif & den Balkan & den Karwendel \\
\hline Génitif & des Balkans & des Karwendels \\
\hline Datif & dem Balkan & dem Karwendel \\
\hline
\end{tabular}

Notons que la flexion constitue un cas privilégié d'appropriation dans la mesure où on plaque sur un exonyme la structure grammaticale de la langue d'accueil. Le français traite ainsi les noms de voies allemands en utilisant invariablement la forme du nominatif (Er wohnt in der Alten Bergstrasse = Il habite dans la Alte Bergstrasse), neutralisant ainsi les flexions casuelles.

À l'inverse, l'allemand ajoute si nécessaire une flexion casuelle à un nom emprunté en altérant par là même la substance de celui-ci (le sommet du Mont Blanc = der Gipfel des Montblancs).

\subsection{Détermination}

La présence d'un article accompagnant le toponyme n'a rien de rare, bien qu'il faille distinguer selon le type de nom propre: peu de noms de villes sont accompagnés d'un article (Den Haag = La Haye) alors qu'il est nécessaire pour tous les noms de cours d'eaux (die Etsch = l'Adige). Notons que la détermination peut être présente en allemand et pas en français et réciproquement.

Sachsen $=\underline{\text { la }}$ Saxe

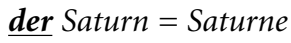

\subsection{Type}

Le rattachement à la typologie Prolex présentée au début de cet article permet non seulement de lever des problèmes de polysémie (Paris \#ville vs Paris \#personnage 
mythique), il permet aussi une tokénisation pertinente (der Roter Main \#hydronyme de forme NPRcomp et non ADJ NPR). Accessoirement, l'indication du type permet une catégorisation utile pour la traduction humaine der Spargel (\#édifice à Francfort), sachant que la définition encyclopédique est souvent superflue pour la compréhension du contexte.

\subsection{Variantes orthographiques}

Malheureusement pour le traitement automatique, le français présente une grande disparité quant à l'orthographe des noms propres composés selon les différents dictionnaires (Mathieu-Colas 1998). La prise en compte des variantes orthographiques est indispensable à toute reconnaissance et en informatique, un caractère en majuscule n'a pas la même valeur qu'un signe en minuscule. Selon les dictionnaires, on trouve indifféremment Place Rouge, place Rouge ou Place rouge.

Certains dictionnaires français vont même jusqu'à utiliser des signes diacritiques inusités en français comme c'est le cas pour Wrocław orthographié dans Le Petit Larousse sur CD-ROM avec un $l$ barré.

Toutefois, l'orthographe peut avoir une valeur discriminante, comme c'est le cas pour le Pas-de-Calais (département français) qui n'est pas le pas de Calais (détroit).

Dans un dictionnaire ou dans une traduction, il faut prendre parti pour une solution cohérente. Notons que l'allemand est, surtout depuis la réforme de l'orthographe, beaucoup plus rigoureux concernant l'utilisation de la majuscule dans les noms composés.

\subsection{Définition sommaire}

Dans une entrée Prolex, la définition n'apparaît pas telle quelle, mais sous forme de relations et d'expansions. Ainsi, dans un texte informatisé en allemand, on risque fort de retrouver Stadt $=$ ville à proximité de Erlau $=$ Eger. Les co-occurrences les plus probables sont listées dans la table des expansions et ce sera pour le type \#ville le mot Stadt en allemand. D'autre part, la ville Erlau est reliée par une relation de méronymie (partie-tout) à Ungarn = Hongrie. À partir de l'expansion et de la relation, il est donc possible de former une définition automatique comme pour die Etsch=l'Adige (cours d'eau d'Italie).

\section{Conclusion}

Les toponymes étrangers sont loin de faire l'objet d'un traitement homogène dans les atlas, les dictionnaires encyclopédiques et les autres supports dans lesquels ils apparaissent. Certes, la tendance est de laisser le toponyme tel quel, semblable à la forme de la langue source, mais il existe de nombreuses exceptions à la fois en synchronie et en diachronie.

\section{NOTES}

1. Présentation des noms locatifs, étude interne au Laboratoire de Linguistique Informatique (UMR 7547 du CNRS).

2. Sur la Toile sous: <http://www.divisionfrancophone.org/>. 
670 MetA, LI, 4, 2006

3. La liste des exonymes publiée par le StAGN est accessible sur la Toile sous: $<$ http://www.bkg.bund. de/Kartographie/Stagn/Exonymenliste.pdf > .

\section{RÉFÉRENCES}

Malblanc, A. (1968): Stylistique comparée du français et de l'allemand, Paris, Didier.

Mathieu-Colas, M. (1998): «La majuscule flottante - Remarques sur l'orthographe des noms propres composés», in: Figement et T.A.L., Besançon, Presses universitaires franc-comtoises, p.123-144.

MeIninger, E. (1986): Dictionnaire des toponymes et des vieux termes mulhousiens, Steinbrunnle-Haut, Éditions du Rhin.

Piton, O., Grass, T. et D. Maurel (2003): "Linguistic resource for NLP. Ask for 'Die Drei Musketiere' and meet 'Les Trois Mousquetaires"', in NLDB 2003 ( $8^{\text {th }}$ International Conference on Applications of Natural Language to Information Systems).

Wydmusch, S. (1998): La toponymie, un patrimoine à préserver, Paris, L'Harmattan. 